\title{
On finite self-complementary metacirculants
}

\author{
Cai Heng Li • Guang Rao • Shu Jiao Song
}

Received: 25 October 2013 / Accepted: 17 March 2014 / Published online: 22 April 2014

(C) Springer Science+Business Media New York 2014

\begin{abstract}
We prove that the automorphism group of a self-complementary metacirculant is either soluble or has $\mathrm{A}_{5}$ as the only insoluble composition factor, extending a result of Li and Praeger which says the automorphism group of a self-complementary circulant is soluble. The proof involves a construction of self-complementary metacirculants which are Cayley graphs and have insoluble automorphism groups. To the best of our knowledge, these are the first examples of self-complementary graphs with this property.
\end{abstract}

Keywords Self-complementary $\cdot$ Vertex-transitive $\cdot$ Metacirculants

\section{Introduction}

A graph $\Gamma=(V, E)$ consists of a vertex set $V$ and an edge set $E$ which is a subset of the set $\{\{u, v\} \mid u, v \in V, u \neq v\}$. The complement $\bar{\Gamma}$ of a graph $\Gamma$ is the graph with the same vertex set $V$ such that $\{u, v\}$ is an edge of $\bar{\Gamma}$ if and only if $u \neq v$, and $\{u, v\}$ is not an edge of $\Gamma$. A graph is said to be self-complementary if its complement is isomorphic to itself.

A graph $\Gamma=(V, E)$ is called a metacirculant if Aut $\Gamma$ contains a metacyclic subgroup $R$ which is transitive on $V$; refer to [16] for the definition which is slightly

C. H. Li · G. Rao $(\varangle)$. S. J. Song

Centre for the Mathematics of Symmetry and Computation, School of Mathematics and Statistics,

The University of Western Australia, Crawley, WA 6009, Australia

e-mail: generalrao@hotmail.com

C. H. Li

e-mail: cai.heng.li@uwa.edu.au

S.J. Song

e-mail: shu-jiao.song@uwa.edu.au 
more general than the original definition of Alspach and Parsons in [2]. (Recall that a group $R$ is called metacyclic if $R$ has a normal subgroup $N$ such that both $N$ and $R / N$ are cyclic.) To emphasise the metacyclic group, we sometimes call $\Gamma$ a metacirculant of $R$.

In this paper, we study metacirculants that are self-complementary, which will be called self-complementary metacirculants.

A special subclass of metacirculants consists of circulants, whose automorphism group contains a transitive cyclic subgroup. Self-complementary circulants have been studied for a long time and have been taken as good models for studying other combinatorial objects, such as Ramsey numbers and communication networks. The first family of self-complementary circulants was constructed by Sachs in 1962, and since then self-complementary circulants have been widely studied, see $[6,19,22,24,25,28]$ for the work till 1980s. In 1990s, the orders of self-complementary circulants were determined, see [1] for Alspach-Morris-Vilfred's proof and [7] for an alternative approach. Some special families of self-complementary circulants are constructed in $[11,18,21]$. More recently, it was proved that the automorphism groups of self-complementary circulants are soluble by Praeger and the first author in [14], and the structure of self-complementary circulants of prime-power order is characterised in [17].

On the other hand, self-complementary metacirculants form a subclass of selfcomplementary vertex-transitive graphs. One of the motivations of studying selfcomplementary vertex-transitive graphs is to investigate Ramsey numbers. For a positive integer $m$, we wish to construct a graph $\Gamma$ with vertex set $V$ such that neither $\Gamma$ nor $\bar{\Gamma}$ contains a complete subgraph $\mathrm{K}_{m}$ and $|V|$ is as big as possible. By intuition, the distribution of pairs of elements of $V$ between $\Gamma$ and $\bar{\Gamma}$ should be "balanced" so that $\Gamma$ and $\bar{\Gamma}$ should not be very different. In the extreme case, $\Gamma \cong \bar{\Gamma}$. Furthermore, since $\mathrm{K}_{m} \nless \Gamma$ is a global property of $\Gamma$, the edges of $\Gamma$ should be distributed "homogeneously". In the ideal case, $\Gamma$ is vertex-transitive. Because of this, self-complementary vertex-transitive graphs have been effectively used as models to find good lower bounds of Ramsey numbers (see [4,5,9,23] for references).

The study of self-complementary vertex-transitive graphs has a long history, refer to the excellent survey of Beezer [3]. In particular, the orders of general selfcomplementary vertex-transitive graphs have been completely determined by Muzychuck [20]. Since 2000, the study of self-complementary vertex-transitive graphs has been advanced by the work in $[10,13]$. The self-complementary vertex-transitive graphs whose orders are products of two primes are classified by the authors in [15].

Recall that for a group $G$ and subgroups $K \triangleleft H \leqslant G$, the quotient group $H / K$ is called a section of $G$. The main result of this paper gives a characterisation of the automorphism groups of self-complementary metacirculants.

Theorem 1.1 Let $\Gamma$ be a self-complementary metacirculant of $R$. Then either Aut $\Gamma$ is soluble or the following hold:

(i) the only insoluble composition factor of Aut $\Gamma$ is the alternating group $\mathrm{A}_{5}$ and

(ii) Aut $\Gamma$ has a section that is of the form $\mathbb{Z}_{p}^{2}:\left(\mathbb{Z}_{\ell} \circ \mathrm{SL}(2,5)\right)$ such that $\mathbb{Z}_{p}^{2}$ is a section of $R$, where $p$ is a prime number and $p \equiv 1$ or $9(\bmod 40)$, and $\ell$ divides $p-1$ and is divisible by 4. 
Remark Although $\mathrm{A}_{5}$ is the only insoluble composition factor of Aut $\Gamma$, this factor can appear multiple times, see Lemma 3.5.

In Sect. 3, we construct examples of self-complementary metacirculants that are Cayley graphs and have insoluble automorphism groups. Moreover, as a result of Lemma 3.3, we have the following.

Theorem 1.2 There exist self-complementary metacirculants of which the automorphism groups are insoluble.

Praeger and the first author in [14] proved that the automorphism group of a selfcomplementary circulant is soluble. A metacirculant $\Gamma$ is called a Sylow-circulant if Aut $\Gamma$ has a transitive metacyclic subgroup $R$ of which all Sylow subgroups are cyclic. In this case, $R$ has a normal Hall subgroup $N$; in particular, the orders of $N$ and $R / N$ are coprime. By Theorem 1.1 (ii), if Aut $\Gamma$ is insoluble, then there exists a prime $p$ such that a Sylow $p$-subgroup of $R$ is not cyclic. Therefore, a consequence of Theorem 1.1 is the following corollary, which extends the result for circulants.

Corollary 1.3 If $\Gamma$ is a self-complementary Sylow-circulant, then Aut $\Gamma$ is soluble. In particular, if $\Gamma$ is a self-complementary metacirculant of square-free order, then Aut $\Gamma$ is soluble.

We end this section with a conjecture.

Conjecture Self-complementary metacirculants are Cayley graphs.

We remark that a metacirculant is generally not necessarily a Cayley graph; for example, the Petersen graph is a metacirculant but not a Cayley graph.

\section{Preliminaries}

In this section, we collect some preliminary results that will be used.

Let $\Gamma=(V, E)$ be a self-complementary graph. Let $\sigma \in \operatorname{Sym}(V)$ be an isomorphism from $\Gamma$ to $\bar{\Gamma}$, called a complementing isomorphism of $\Gamma$. Then $\sigma$ interchanges $\Gamma$ and $\bar{\Gamma}$, and $\sigma$ has order $o(\sigma)=2^{e} b$ for $e \geqslant 1$, where $b$ is an odd number. Observe that $\sigma=\sigma^{b} \cdot \sigma^{1-b}$, where $\sigma^{b}$ is a complementing isomorphism of 2-power order and $\sigma^{1-b} \in$ Aut $\Gamma$. We thus sometimes assume a complementing isomorphism $\sigma$ to be of 2-power order. If $o(\sigma)=2$, then $\sigma$ interchanges some vertices $u, v$ and fixes $\{u, v\}$, which is not possible. So 4 divides $o(\sigma)$.

Lemma 2.1 A complementing isomorphism of a self-complementary graph has order divisible by 4.

For a regular self-complementary graph of order $n$, a vertex has an equal number of neighbours and non-neighbours, and hence $n$ must be an odd number. Furthermore, the graph has precisely half number of edges of the complete graph, and so $n(n-1) / 4$ is an integer, and $n \equiv 1(\bmod 4)$. 
Lemma 2.2 The order of a regular self-complementary graph is congruent to 1 (modulo 4).

We next introduce a classical method for constructing self-complementary graphs. For a finite group $R$, let $R^{\#}:=R \backslash\{1\}$, the set of all non-identity elements of $R$. For a subset $S \subseteq R^{\#}$ with $S=S^{-1}:=\left\{s^{-1} \mid s \in S\right\}$, the associated Cayley graph Cay $(R, S)$ is the graph with vertex set $V=R$ and edge set $E=\{\{a, b\} \mid a, b \in$ $\left.R, b a^{-1} \in S\right\}$.

By the definition, the Cayley graph Cay $\left(R, R^{\#} \backslash S\right)$ is the complement of Cay $(R, S)$. Observe that any automorphism $\sigma \in \operatorname{Aut}(R)$ induces an isomorphism from Cay $(R, S)$ to $\operatorname{Cay}\left(R, S^{\sigma}\right)$. Thus, if there exist $S \subseteq R^{\#}$ and $\sigma \in \operatorname{Aut}(R)$ such that

$$
S^{\sigma}=R^{\#} \backslash S
$$

then $\operatorname{Cay}(R, S) \cong \operatorname{Cay}\left(R, S^{\sigma}\right)=\operatorname{Cay}\left(R, R^{\#} \backslash S\right)$ is self-complementary. Since $S$ and $R^{\#} \backslash S$ are disjoint, $\sigma$ fixes no non-identity element of $R$, that is, $\sigma$ is a fixed-point-free automorphism of $R$. Moreover, since $S=S^{-1}$, and $\sigma$ cannot interchange any pair of vertices, we further have that $\sigma^{2}$ is fixed-point-free, and $g^{-1} \in g^{\left\langle\sigma^{2}\right\rangle}$ for any $g \in R$.

Conversely, if a group $R$ has an automorphism $\sigma$ of order a power of 2 such that $\sigma^{2}$ is fixed-point-free, then the following construction indeed produces self-complementary graphs, see [12].

Construction 2.3 Let $R$ be a group and let $\sigma \in \operatorname{Aut}(R)$ of order a power of 2 such that $\sigma^{2}$ is fixed-point-free. Then every orbit of $\langle\sigma\rangle$ on $R^{\#}$ has length divisible by 4 and is divided into two parts by $\sigma^{2}$. We define a subset $S$ and a Cayley graph Cay $(R, S)$ as follows:

(1) Let $\Delta_{1}, \Delta_{2}, \ldots, \Delta_{r}$ be the $\langle\sigma\rangle$-orbits on $R^{\#}$, and label the two orbits of $\left\langle\sigma^{2}\right\rangle$ on $\Delta_{i}$ as $\Delta_{i}^{+}$and $\Delta_{i}^{-}$, where $1 \leqslant i \leqslant r$.

(2) Set $S=\bigcup_{i=1}^{r} \Delta_{i}^{\varepsilon_{i}}$, where $\varepsilon_{i}=+$ or - . (We remark that there are $2^{r}$ different choices for such a subset $S$.)

Another construction method of self-complementary vertex-transitive graphs is based on the lexicographic product (sometimes called the wreath product). Let $\Gamma_{1}=$ $\left(V_{1}, E_{1}\right)$ and $\Gamma_{2}=\left(V_{2}, E_{2}\right)$ be graphs. The lexicographic product of $\Gamma_{2}$ by $\Gamma_{1}$ is defined as the graph with vertex set $V=V_{1} \times V_{2}$ such that $\left(u_{1}, v_{1}\right)$ is adjacent to $\left(u_{2}, v_{2}\right)$ if and only if either $u_{1}$ is adjacent to $u_{2}$ in $\Gamma_{1}$ or $u_{1}=u_{2}$ and $v_{1}$ is adjacent to $v_{2}$. This graph is denoted by $\Gamma_{1}\left[\Gamma_{2}\right]$.

The lexicographic product has the following nice properties (see [3]):

Lemma 2.4 If both $\Gamma_{1}, \Gamma_{2}$ are vertex-transitive or self-complementary, then so is $\Gamma_{1}\left[\Gamma_{2}\right]$.

We will also need to know about the order of the maximal $p$-elements in $\operatorname{GL}(d, p)$.

Lemma 2.5 If $d \geqslant 2$, then the largest order $p^{e}$ of $p$-elements of $\mathrm{GL}(d, p)$ satisfies $p^{e} \geqslant d>p^{e-1}$. 
Proof Let $U$ be the set of the elements of $\operatorname{GL}(d, p)$ of the following form:

$$
\left(\begin{array}{ccccc}
1 & a_{12} & a_{13} & \cdots & a_{1 d} \\
0 & 1 & a_{23} & & a_{2 d} \\
\vdots & 0 & \ddots & \ddots & \vdots \\
\vdots & \vdots & \ddots & \ddots & a_{d-1, d} \\
0 & 0 & \cdots & 0 & 1
\end{array}\right) .
$$

Then $U$ forms a Sylow $p$-subgroup of $\operatorname{GL}(d, p)$. Let $u \in U$ such that $o(u)=p^{e}$ is the largest order of elements of $U$, where $e$ is an integer. Let $u=A+I$. Notice that for any $u \in U$, we have $u^{p^{e}}=I$ if and only if $(A+I)^{p^{e}}=I$, if and only if (using the binomial theorem) $A^{p^{e}}=0$. By careful choice of $u$, we can ensure that $A^{b} \neq 0$ for $b<d$, so that $A^{p^{e}}=0$ implies $p^{e} \geqslant d$ and $p^{e-1}<d$.

\section{Examples with insoluble automorphism groups}

We construct examples of self-complementary metacirculants which have insoluble automorphism groups.

Let $R=\mathbb{Z}_{p}^{2}$ with $p$ prime. Then $\operatorname{Aut}(R)=\mathrm{GL}(2, p)$. Assume that $p \equiv$ $\pm 1(\bmod 10)$. Then $\operatorname{GL}(2, p)$ contains an irreducible subgroup $\operatorname{SL}(2,5)$, see [26, page 411$]$. Assume further that $p \equiv 1(\bmod 8)$. Then the centre $\mathbf{Z}(\operatorname{GL}(2, p)) \cong \mathbb{Z}_{p-1}$ has order divisible by 8 . Let $\sigma$ be a 2-element of $\mathbf{Z}(\operatorname{GL}(2, p))$ which has order at least 8. Since $\sigma$ is a scalar matrix, its action on $R$ is fixed-point-free. Then $\langle\sigma, \operatorname{SL}(2,5)\rangle=\langle\sigma\rangle \circ \operatorname{SL}(2,5)$ is a subgroup of $\operatorname{GL}(2, p)$. We note that the condition that $p \equiv \pm 1(\bmod 10)$ and $p \equiv 1(\bmod 8)$ is equivalent to $p \equiv 1$ or $9(\bmod 40)$.

Lemma 3.1 Let $M=\left\langle\sigma^{2}, \operatorname{SL}(2,5)\right\rangle$ and $H=\langle\sigma, \operatorname{SL}(2,5)\rangle$. Then $M$ divides each $H$-orbit on $R^{\#}$ into two parts of equal size.

Proof Let $\Delta$ be an $H$-orbit on $R^{\#}$, and let $x \in \Delta$. Then $x^{\sigma} \in \Delta$. Suppose that $M$ is transitive on $\Delta$. Then there exists an element $g \in M$ such that $x^{g}=x^{\sigma}$. Thus, $x^{\sigma g^{-1}}=x$. Since $\sigma$ is in the centre of $H, \sigma g^{-1}=g^{-1} \sigma$. Hence $\left(\sigma g^{-1}\right)^{o(g)}=$ $\sigma^{o(g)}\left(g^{-1}\right)^{o(g)}=\sigma^{o(g)}$. As no element of $\operatorname{SL}(2,5)$ has order divisible by 8 , the power $\sigma^{o(g)}$ is not the identity. Since $\sigma g^{-1}$ fixes $x$, so does the power $\left(\sigma g^{-1}\right)^{o(g)}=\sigma^{o(g)}$. This is not possible since the scalar $\sigma^{o(g)}$ is a fixed-point-free automorphism of $R$. Therefore, $M$ is not transitive on $\Delta$. Since $M$ is a normal subgroup of $H$ of index 2, $M$ acts on $\Delta$ half-transitively and has exactly two equal size orbits.

This lemma enables us to have the following construction.

Construction 3.2 Using the notation above, we define a graph as below.

(1) Let $\Delta_{1}, \ldots, \Delta_{r}$ be the orbits of $H$ acting on $R^{\#}$, and let $\Delta_{i}^{+}$and $\Delta_{i}^{-}$be the two orbits of $M$ on $\Delta_{i}$, where $1 \leqslant i \leqslant r$.

(2) Let $S=\bigcup_{i=1}^{r} \Delta_{i}^{\varepsilon_{i}}$, where $\varepsilon_{i}=+$ or - , and let $\Gamma=\operatorname{Cay}(R, S)$. 
Lemma 3.3 Let $\Gamma$ be a Cayley graph constructed in Construction 3.2. Then $\Gamma$ is a self-complementary metacirculant with insoluble automorphism group Aut $\Gamma \geqslant$ $\mathbb{Z}_{p}^{2}:\left(\left\langle\sigma^{2}\right\rangle \circ \mathrm{SL}(2,5)\right)$, and $\sigma$ is a complementing isomorphism.

Proof By definition, $\sigma$ interchanges $\Delta_{i}^{+}$and $\Delta_{i}^{-}$, where $1 \leqslant i \leqslant r$. Thus,

$$
S^{\sigma}=\bigcup_{i=1}^{r}\left(\Delta_{i}^{\varepsilon_{i}}\right)^{\sigma}=\bigcup_{i=1}^{r} \Delta_{i}^{-\varepsilon_{i}}=R^{\#} \backslash S .
$$

Since $\sigma$ is a scalar of order divisible by 8 , the power $\sigma^{o(\sigma) / 2}$ maps every element $x \in R$ into the inverse $x^{-1}$. Hence each $\Delta_{i}$ is self-inverse and so is $S$. So $\Gamma$ is undirected and is self-complementary with $\sigma$ being a complementing isomorphism.

Since $R$ is metacyclic, the Cayley graph $\Gamma$ of $R$ is a metacirculant. Moreover, Aut $\Gamma \geqslant R: M=\mathbb{Z}_{p}^{2}:\left\langle\sigma^{2}, \operatorname{SL}(2,5)\right\rangle=\mathbb{Z}_{p}^{2}:\left(\left\langle\sigma^{2}\right\rangle \circ \operatorname{SL}(2,5)\right)$ is insoluble.

By iteratively taking the lexicographic products of graphs constructed via Construction 3.2, we can in fact construct self-complementary metacirculants with insoluble automorphism groups, which are Cayley graphs on $\mathbb{Z}_{n_{1} n_{2}}^{2}$ for any coprime pair $n_{1}, n_{2}$ of square-free integers. Construction 6.1 and Lemma 6.2 of [8] show that the resulting graphs are in fact self-complementary metacirculants with insoluble automorphism groups in which the insoluble composition factor $\mathrm{A}_{5}$ appears multiple times.

We next give a different construction of self-complementary metacirculants of which the automorphism groups contain a section $\mathrm{A}_{5} \times \cdots \times \mathrm{A}_{5}$.

Let $p_{1}, \ldots, p_{t}$ be distinct primes such that $p_{i} \equiv 1$ or $9(\bmod 40)$ for each $i \in$ $\{1, \ldots, t\}$. Let $R_{i}=\mathbb{Z}_{p_{i}}^{2}$, and let $L_{i}<\operatorname{Aut}\left(R_{i}\right)=\mathrm{GL}\left(2, p_{i}\right)$ be isomorphic to $\operatorname{SL}(2,5)$. Let $\sigma_{i} \in \mathbf{Z}\left(\right.$ Aut $\left.\left(R_{i}\right)\right)$ have order divisible by 8 . Let $R=R_{1} \times \cdots \times R_{t}$, $L=L_{1} \times \cdots \times L_{t}$, and $\sigma=\left(\sigma_{1}, \ldots, \sigma_{t}\right) \in \operatorname{Aut}(R)$. Let $H=R:(\langle\sigma\rangle \circ L)$ and $M=R:\left(\left\langle\sigma^{2}\right\rangle \circ L\right)$.

Lemma 3.4 The subgroup $M$ divides every $H$-orbit on $R^{\#}$ into two parts of equal size.

Proof (The proof is similar to the proof of Lemma 3.1.) Let $\Delta$ be an $H$-orbit on $R^{\#}$, and let $\left(x_{1}, \ldots, x_{t}\right) \in \Delta$. Suppose that there is an element $g \in M$ such that $\left(x_{1}, \ldots, x_{t}\right)^{g}=\left(x_{1}, \ldots, x_{t}\right)^{\sigma}$. Then $\left(x_{1}, \ldots, x_{t}\right)^{\sigma g^{-1}}=\left(x_{1}, \ldots, x_{t}\right)$. As $\sigma g^{-1}=$ $g^{-1} \sigma$, we have $\left(\sigma g^{-1}\right)^{o(g)}=\sigma^{o(g)}\left(g^{-1}\right)^{o(g)}=\sigma^{o(g)}$. Noticing that no element of $L$ has order divisible by 8 , we know $\sigma^{o(g)} \neq 1$. Since $\sigma g^{-1}$ fixes $\left(x_{1}, \ldots, x_{t}\right)$, so does the power $\sigma^{o(g)}$. Now some $x_{i} \neq 1$, and $x_{i}^{\sigma_{i}^{o(g)}}=x_{i}$, which contradicts that the scalar $\sigma_{i}^{o(g)}$ is a fixed-point-free automorphism of $R_{i}$. So $M$ is intransitive on $\Delta$ and has exactly two orbits of equal size.

Thus, applying Construction 3.2 with $H, M$ defined before Lemma 3.4 produces examples of self-complementary metacirculants of which the automorphism groups have a section $\mathrm{A}_{5}^{t}$ for any positive integer $t$.

Lemma 3.5 Let $p_{1}, \ldots, p_{t}$ be distinct primes such that $p_{i} \equiv 1$ or $9(\bmod 40)$ for every $i \in\{1, \ldots, t\}$. Then there exist self-complementary metacirculants $\Gamma$ of order 
$p_{1}^{2} \ldots p_{t}^{2}$ such that Aut $\Gamma \geqslant \mathbb{Z}_{p_{1} \ldots p_{t}}^{2}:\left(\mathbb{Z}_{\ell} \circ \operatorname{SL}(2,5)^{t}\right)$, where $\ell \mid\left(p_{i}-1\right)$ for each $i$ and $8 \mid \ell$.

\section{Proof of Theorem 1.1}

Let $\Gamma=(V, E)$ be a self-complementary metacirculant of a metacyclic group $R$. By [14], we may assume that $R$ is not cyclic. Let $G=$ Aut $\Gamma$, and let $\sigma$ be a complementing isomorphism of $\Gamma$. Let $X=\langle G, \sigma\rangle$. Then $\sigma^{2} \in G$, and $X=G . \mathbb{Z}_{2}$. Let $n$ be a positive integer, and let $p$ be a prime factor of $n$. By $n_{p}$ we denote the highest power of $p$ that divides $n$, so that $p$ and $n / n_{p}$ are coprime.

\subsection{The primitive case}

We consider first the vertex-primitive case. Suppose that $X$ is primitive on $V$. Then a result of [10] states that either $X$ is affine or the $\operatorname{socle} \operatorname{soc}(X)=\operatorname{PSL}\left(2, q^{2}\right)^{\ell}$ and $|V|=\left(\frac{1}{2} q^{2}\left(q^{2}+1\right)\right)^{\ell}$, where $q$ is odd and $\ell \geqslant 2$. We first exclude the latter case.

Lemma 4.1 The group $X$ is affine.

Proof Suppose that $X$ has socle $N=\operatorname{PSL}\left(2, q^{2}\right)^{\ell}$, and $|V|=\left(\frac{1}{2} q^{2}\left(q^{2}+1\right)\right)^{\ell}$. Since $R$ is transitive on $V$, it follows that $q^{2 \ell}$ divides $|R|$. Let $q=p^{f}$ with $p$ prime and $f \geqslant 1$. Since $R$ is metacyclic, $R$ contains an element $x$ of order $q^{\ell}=p^{f \ell}$. Let $K$ be the largest subgroup of $X$ which normalises each direct factor of $N$. Then $N \triangleleft K \leqslant \operatorname{P\Gamma L}\left(2, q^{2}\right)^{\ell}$. Note that $\operatorname{P\Gamma L}\left(2, q^{2}\right)=\operatorname{PGL}\left(2, q^{2}\right): \mathbb{Z}_{2 f}$, and $\operatorname{PGL}\left(2, q^{2}\right)$ has a Sylow $p$-subgroup $\mathbb{Z}_{p}^{2 f}$. Hence a $p$-element of $K$ has order at most $p f_{p}$. Thus,

$$
X / K \geqslant\langle x\rangle K / K \cong\langle x\rangle /(\langle x\rangle \cap K) \text {, }
$$

and so $X / K \leqslant S_{\ell}$ contains an element of order $p^{f \ell-1} / f_{p}$. However, $p^{f \ell-1} / f_{p}$ does not divide $\left|S_{\ell}\right|=\ell$ !, which is a contradiction.

We now show that only affine groups of dimension 2 can appear.

\section{Lemma 4.2 The primitive group $X$ is affine of dimension 2 .}

Proof By Lemma 4.1 the socle $N=\operatorname{soc}(X)=\mathbb{Z}_{p}^{d}$, where $p$ is a prime and $d \geqslant 1$. Then $X \leqslant \operatorname{AGL}(d, p)$, and $p^{d}$ divides $|R|$. Since $R$ is metacyclic, there exists an element $g \in R$ of order divisible by $p^{\left\lceil\frac{d}{2}\right\rceil}$. Since $\operatorname{AGL}(d, p)<\operatorname{GL}(d+1, p)$ and they have isomorphic Sylow $p$-subgroups, by Lemma 2.5, the largest order $p^{e}$ of the $p$ elements of $\operatorname{AGL}(d, p)$ is such that $p^{e} \geqslant d+1>p^{e-1}$. Thus, $p^{\left\lceil\frac{d}{2}\right\rceil-1} \leqslant p^{e-1}<d+1$. Noticing that $p \geqslant 3$, this implies that $d \leqslant 4$.

Suppose that $d=3$. Then $p^{\left\lceil\frac{d}{2}\right\rceil}=p^{2} \leqslant p^{e}$, and $p \leqslant p^{e-1}<d+1=4$. Thus $p=3$, and $|V|=3^{3} \not \equiv 1(\bmod 4)$, which is not possible by Lemma 2.2 .

Suppose that $d=4$. Then $\left\lceil\frac{d}{2}\right\rceil=2$, and it follows that $p \leqslant p^{e-1}<d+1=5$. Thus $p=3$, and $|V|=3^{4}$. Without loss of generality, we may assume that $R$ is a 
3-group. Note that AGL $(4,3)<\operatorname{GL}(5,3)$, by Lemma 2.5, the largest order of the 3elements of $\operatorname{GL}(5,3)$ is 9 . Thus, the metacyclic group $R=\mathbb{Z}_{9} \cdot \mathbb{Z}_{9}$. However, checking the subgroups of $\operatorname{GL}(5,3)$ by GAP [27] leads to the conclusion that $\operatorname{GL}(5,3)$ does not have a metacyclic group $\mathbb{Z}_{9} \cdot \mathbb{Z}_{9}$.

Finally, since we have assumed that $R$ is not cyclic, we conclude that $d=2$.

Next, we determine the insoluble case.

Lemma 4.3 Assume that $X$ is primitive and insoluble. Then we have

$$
X=\mathbb{Z}_{p}^{2}:\left(\mathbb{Z}_{\ell} \circ \operatorname{SL}(2,5)\right), \text { and } G=\mathbb{Z}_{p}^{2}:\left(\mathbb{Z}_{\ell / 2} \circ \operatorname{SL}(2,5)\right)
$$

where $p \equiv 1$ or $9(\bmod 40)$, and $\ell$ divides $p-1$ and is divisible by 8 .

Proof Since $X$ is primitive and insoluble, by Lemma 4.2, we have $|V|=p^{2}$, and $X \leqslant \operatorname{AGL}(2, p)$, and the stabiliser $X_{v}$ is an irreducible subgroup of $\operatorname{GL}(2, p)$.

Notice that $G$ has index 2 in $X$ and is not 2-transitive on $V$, so $G_{v}$ does not contain $\operatorname{SL}(2, p)$. Inspecting the subgroups of $\operatorname{SL}(2, p)$ listed in [26, Theorem 6.17], we conclude that $X_{v}$ contains a subgroup isomorphic to $\operatorname{SL}(2,5)$ with $p=5$ or $p \equiv \pm 1(\bmod 5)$. Noting that $\mathbb{Z}_{p-1} \circ \mathrm{SL}(2,5)$ is maximal in $\mathbb{Z}_{p-1} \circ \mathrm{SL}(2, p)$ and there is no element of $\operatorname{GL}(2, p) \backslash\left(\mathbb{Z}_{p-1} \circ \mathrm{SL}(2,5)\right)$ which normalises $\operatorname{SL}(2,5)$, we have

$$
X_{v}=\langle z\rangle \circ \operatorname{SL}(2,5)=\mathbb{Z}_{\ell} \circ \operatorname{SL}(2,5),
$$

where $z$ is a scalar of order $\ell$ dividing $p-1$. Since $G$ is transitive on $V$, the index $\left|X_{v}: G_{v}\right|=|X: G|=2$. We may assume $\sigma \in X_{v}$. As SL(2, 5) has no subgroup of index $2, \sigma \notin \operatorname{SL}(2,5)$. Since $\sigma$ normalises $G_{v}, \sigma$ normalises $\operatorname{SL}(2,5)$, and we may choose $\sigma \in\langle z\rangle$. Then $\sigma \notin\left\langle z^{2}\right\rangle$, and $G_{v}=\left\langle z^{2}\right\rangle \circ \operatorname{SL}(2,5)$. By Lemma 2.1, the order $o(\sigma)$ is divisible by 4 .

In particular, the minimal normal subgroup $\mathbb{Z}_{p}^{2}$ of $X$ is regular on $V$ and is a Sylow $p$-subgroup. So $\Gamma$ is a metacirculant and a Cayley graph of $R$, say

$$
\Gamma=\operatorname{Cay}(R, S) \text {. }
$$

Suppose that $\ell$ is not divisible by 8 . Then since $\sigma \in \mathbb{Z}_{\ell}, \sigma$ is of order $4 m$ with $m$ odd, and

$$
X_{v}=\left\langle\sigma^{4}\right\rangle \times\left(\mathbb{Z}_{4} \circ \mathrm{SL}(2,5)\right)=\mathbb{Z}_{m} \times\left(\mathbb{Z}_{4} \circ \operatorname{SL}(2,5)\right),
$$

and $G_{v}=\mathbb{Z}_{m} \times \operatorname{SL}(2,5)$. Let $\tau=\sigma^{m} \in\langle z\rangle$. Then $\tau$ is of order 4 , and $X_{v}=\langle\tau\rangle \circ G_{v}$. For every element $x \in R$, we have $x^{\tau}=x^{j}$ for some integer $j$ which has order 4 in the multiplicative group $\mathrm{GF}(p)^{*}$ of $\mathrm{GF}(p)$.

Let $y \in G_{v}$ be of order 4 . Since 4 divides $p-1$, the group $\langle y\rangle$ is reducible on $R=\operatorname{soc}(X)$. Thus, $y$ normalises a cyclic subgroup $\langle w\rangle \cong \mathbb{Z}_{p}$, where $w \in R$, and $y$ induces an automorphism of $\langle w\rangle$ of order 4. So $w^{y}=w^{k}$, for some integer $k$ which has order 4 in $\mathrm{GF}(p)^{*}$. Since $\mathrm{GF}(p)^{*}$ has a unique subgroup of order $4, k=j$ or $j^{-1}$ in $\operatorname{GF}(p)^{*}$. Thus, $w^{\tau}=w^{y}$ or $w^{y^{-1}}$, respectively. Without loss of generality, assume 
that $w \in S$. Since $y \in G$, both $w^{y}$ and $w^{y^{-1}}$ belong to $S$. However, $w^{\tau} \in S^{\tau}=R^{\#} \backslash S$, which is a contradiction. Hence 8 divides $o(\sigma)$, and $p \equiv 1(\bmod 8)$.

Finally, since $p \equiv \pm 1(\bmod 5)$, it follows that $p \equiv 1$ or $9(\bmod 40)$. The proof is completed.

\subsection{Proof of Theorem 1.1}

In order to prove Theorem 1.1, we assume that $X$ is insoluble. We complete our proof by induction on the order $|V|$.

If $X$ is primitive on $V$, then by Lemmas 4.2 and 4.3, the statement of Theorem 1.1 is true. We thus assume that $X$ is imprimitive on $V$, and further assume that Theorem 1.1 holds for any self-complementary metacirculant of order properly dividing $|V|$.

Let $\mathcal{B}$ be an $X$-invariant partition of $V$, called a block system of $X$ on $V$. Each element $B \in \mathcal{B}$ is called a block. By $[B]_{\Gamma}$ we denote the induced subgraph of $\Gamma$ on $B$. We need the following result (see [13]).

Theorem 4.4 (Li-Praeger 2003) Suppose that $V$ has a block system $\mathcal{B}$ under the action of $X$. Then the following hold:

(i) for each $B \in \mathcal{B}$, the induced subgraph $[B]_{\Gamma}$ is self-complementary, $G_{B}^{B} \leqslant$ Aut $[B]_{\Gamma}$, and $\sigma$ induces a complementing isomorphism of $[B]_{\Gamma}$;

(ii) there is a self-complementary graph $\Sigma$ with vertex set $\mathcal{B}$ such that $G^{\mathcal{B}} \leqslant$ Aut $\Sigma$ and any element of $X^{\mathcal{B}} \backslash G^{\mathcal{B}}$ is a complementing isomorphism of $\Sigma$.

Let $K=X_{(\mathcal{B})}$ be the kernel of $X$ acting on $\mathcal{B}$. Then, as $|\mathcal{B}|$ properly divides $|V|$, by our assumption, Theorem 1.1 holds for $X^{\mathcal{B}}$, that is, if $X^{\mathcal{B}}$ is insoluble, then $\mathrm{A}_{5}$ is the only insoluble composition factor of $X^{\mathcal{B}}$, and $X^{\mathcal{B}}$ has a section which has the form

$$
\mathbb{Z}_{q}^{2}:\left(\mathbb{Z}_{m} \circ \operatorname{SL}(2,5)\right)
$$

where $q$ is a prime, $8 \mid m$ and $m \mid(q-1)$ and $\mathbb{Z}_{q}^{2}$ is a section of $R^{\mathcal{B}}$.

If $K$ is soluble, then $X=K . X^{\mathcal{B}}$ satisfies Theorem 1.1. We thus assume that $K$ is insoluble. Then $K^{B}$ is insoluble and so is $X_{B}^{B}$.

To complete the proof, we may further assume that $\mathcal{B}$ is a minimal block system of $X$, that is, for a block $B \in \mathcal{B}$, the induced action $X_{B}^{B}$ is primitive. By Lemmas 4.2 and 4.3, we have that $X_{B}^{B}$ is affine of degree $p$ or $p^{2}$ with $p$ prime, and as $X_{B}^{B}$ is insoluble,

$$
X_{B}^{B}=\mathbb{Z}_{p}^{2}:\left(\mathbb{Z}_{\ell} \circ \operatorname{SL}(2,5)\right)
$$

and $R_{B}^{B}=\mathbb{Z}_{p}^{2}$, where $p \equiv 1$ or $9(\bmod 40)$ is a prime, as in part (ii) of Theorem 1.1.

Since $K \triangleleft X_{B}$ is insoluble, $K^{B}=\mathbb{Z}_{p}^{2}:\left(\mathbb{Z}_{\ell^{\prime}} \circ \operatorname{SL}(2,5)\right)$, where $\ell^{\prime} \mid \ell$. Furthermore, since $K \leqslant K^{B_{1}} \times \cdots \times K^{B_{t}}$, where $\mathcal{B}=\left\{B_{1}, \ldots, B_{t}\right\}$, we conclude that $\mathrm{A}_{5}$ is the only insoluble composition factor of $K$. Finally, since $X=K . X^{\mathcal{B}}$ is an extension of $K$ by $X^{\mathcal{B}}$, every composition factor of $X$ is a composition factor of $K$ or $X^{\mathcal{B}}$. Therefore, $\mathrm{A}_{5}$ is the only insoluble composition factor of $X$. This completes the proof of our theorem. 
Acknowledgments This work was partially supported by an ARC Discovery Project grant.

\section{References}

1. Alspach, B., Morris, J., Vilfred, V.: Self-complementary circulant graphs. Ars Combin. 53, 187-191 (1999)

2. Alspach, B., Parsons, T.D.: A construction for vertex-transitive graphs. Canad. J. Math. 34, 307-318 (1982)

3. Beezer, R.A.: Sylow subgraphs in self-complementary vertex transitive graphs. Expo. Math. 24(2), 185-194 (2006)

4. Chvátal, V., Erdös, P., Hedrlín, Z.: Ramsey's theorem and self-complementary graphs. Disc. Math. 3, 301-304 (1972)

5. Clapham, C.R.J.: A class of self-complementary graphs and lower bounds of some Ramsey numbers. J. Graph Theory 3(3), 287-289 (1979)

6. Figueroa, R., Giudici, R.E.: Group generation of self-complementary graphs. In: Combinatorics and Graph Theory (Hefei, 1992), pp. 131-140. World Science Publisher, River Edge, NJ (1993)

7. Fronček, D., Rosa, A., Širán̆, J.: The existence of self-complementary circulant graphs. Eur. J. Combin. 17(7), 625-628 (1996)

8. Giudici, M., Li, C.H., Potoncik, P., Praeger, C.E.: Homogeneous factorisations of graph products. Disc. Math. 308(16), 3652-3667 (2008)

9. Gulden, F., Tomasta, P.: New lower bounds of some diagonal Ramsey numbers. J. Graph Theory 7(1), 149-151 (1983)

10. Guralnick, R., Li, C.H., Praeger, C.E., Saxl, J.: On orbital partitions and exceptionality of primitive permutation groups. Trans. Am. Math. Soc. 356(12), 4857-4872 (2004)

11. Jajcay, R., Li, C.H.: Constructions of self-complementary circulants with no multiplicative isomorphisms. Eur. J. Combin. 22(8), 1093-1100 (2001)

12. Li, C.H.: On finite graphs that are self-complementary and vertex-transitive. Australas. J. Combin. 18, 147-155 (1998)

13. Li, C.H., Praeger, C.E.: On partitioning the orbitals of a transitive permutation group. Trans. Am. Math. Soc. 355(2), 637-653 (2003)

14. Li, C.H., Praeger, C.E.: On finite permutation groups with a transitive cyclic subgroup. J. Algebra $\mathbf{3 4 9}$, 117-127 (2012)

15. Li, C. H., and Rao, G.: Self-complementary vertex-transitive graphs of order a product of two primes. Bull. Austral. Math. Soc. 89(2), 322-330 (2014)

16. Li, C.H., Song, S.J., Wang, D.J.: A characterization of metacirculants. J. Combin. Theory Ser. A 120(1), 39-48 (2013)

17. Li, C.H., Sun, S.H., Xu, Jing: Self-complementary circulants of prime-power order. Siam J. Disc. Math. 28(1), 8-17 (2014)

18. Liskovets, V., Poschel, R.: Non-Cayley-isomorphic self-complementary circulant graphs. J. Graph Theory 34(2), 128-141 (2000)

19. Mathon, R.: On self-complementary strongly regular graphs. Disc. Math. 69(3), 263-281 (1988)

20. Muzychuck, M.: On Sylow subgraphs of vertex-transitive self-complementary graphs. Bull. Lond. Math. Soc. 31(5), 531-533 (1999)

21. Praeger, C.E., Li, C.H., Stringer, L.: Common circulant homogeneous factorisations of complete graphs. Disc. Math. 309(10), 3006-3012 (2009)

22. Rao, S.B.: On regular and strongly regular self-complementary graphs. Disc. Math. 54(1), 73-82 (1985)

23. Rödl, V., Šinajová, E.: Note on Ramsey numbers and self-complementary graphs. Math. Slovaca 45(3), 243-249 (1995)

24. Sachs, H.: Über selbstcomplementäre graphen. Publ. Math. Debrecen 9, 270-288 (1962)

25. Suprunenko, D.A.: Self-complementary graphs. Cybernetics 21(5), 559-567 (1985)

26. Suzuki, M.: Group Theory I. Springer-Verlag, New York (1982)

27. The GAP Group, GAP - Groups, Algorithms, and Programming, Version 4.3 (The GAP Group, Aachen/St Andrews, 2002); http://www-gap.dcs.st-and.ac.uk

28. Zelinka, B.: Self-complementary vertex-transitive undirected graphs. Math. Slovaca 29(1), 91-95 (1979) 